\title{
A Sub-arcsecond Study of Ionized Gas in NGC 253
}

\author{
K.R. Anantharamaiah \& Niruj R. Mohan ${ }^{\dagger}$ \\ Raman Research Institute, Sadhashivanagar, Bangalore $560080{ }^{\dagger}$ and \\ Dept. of Physics, Indian Institute of Science, Bangalore 560012, India
}

\author{
W.M. Goss
}

NRAO, P.O. Box O, Socorro, NM 87801, USA

\begin{abstract}
.
The nuclear region of the starburst galaxy NGC 253 was observed in $\mathrm{H} 92 \alpha(8.3 \mathrm{GHz})$ and $\mathrm{H} 75 \alpha(15.4 \mathrm{GHz})$ recombination lines at subarcsecond resolution using the A-configuration of the Very Large Array (VLA). In the highest resolution observations at $15.3 \mathrm{GHz}$, we have detected a compact $(<5 \mathrm{pc})$, high density $\left(>5 \times 10^{4} \mathrm{~cm}^{-3}\right)$ ionized region which is nearly coincident with the radio continuum peak near the nucleus. Modeling of RRL emission from this region shows that about 1000 $\mathrm{O}$ stars are needed to maintain the ionization. Assuming the Salpeter initial mass function, the implied overall stellar density corresponds to that of a super star cluster.
\end{abstract}

\section{Introduction}

Ionized gas in the nuclear region of the prototypical starburst galaxy NGC 253 has been studied at arcsecond resolutions at optical (Arnaboldi et al 1995, Prada, Gutierrez, \& McKeith 1998), infrared (Engelbracht et al 1998, Boker, Krabbe, \& Storey 1998, Keto et al 1999) and radio (Anantharamaiah \& Goss 1996) wavelengths. These studies have revealed the presence of large quantities of ionized gas in the central $100 \mathrm{pc}$ region with complex spatial and kinematic structures. Radio continuum observations at sub-arcsecond resolution (Ulvestad \& Antonucci 1997) have shown a number of compact sources within the central $15^{\prime \prime}$ region including a bright source near the possible nucleus. High resolution optical observations with the Hubble Space Telescope have discovered four super star clusters (SSCs) in the central region (Watson et al 1996). None of the SSCs have a positional correspondence with the radio continuum peaks. The brightest peak observed in mid-infrared images at arcsecond resolution (Boker et al 1998, Keto et al 1999) is also not coincident with the central compact radio source but is offset by about $2.5^{\prime \prime}$ to the southeast. The $12.8 \mu \mathrm{m}$ [Ne II] emission peak at this offset position has been interpreted by Keto et al (1999) as ionized gas excited by OB stars of one of the SSCs discovered by Watson et al (1996). SSCs, which have been found in many starburst regions (Meurer et al 1995) are believed to be progenitors of the present day globular clusters. 

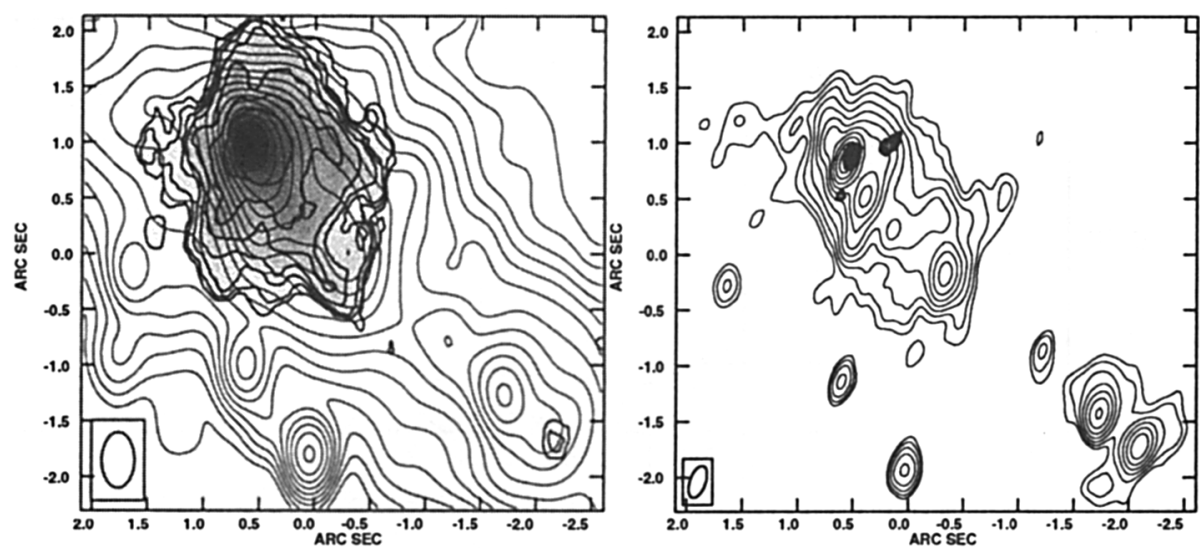

Figure 1. Radio Continuum (thin contours) and velocity-integrated recombination line emission (thick contours and grey scale) at $3.6 \mathrm{~cm}$ (left) and $2 \mathrm{~cm}$ (right). The beams, shown as insets, are $0.5^{\prime \prime} \times 0.3^{\prime \prime}$, $\mathrm{PA}=0^{\circ}$ at $3.6 \mathrm{~cm}$ and $0.3^{\prime \prime} \times 0.15^{\prime \prime}, \mathrm{PA}=-20^{\circ}$ at $2 \mathrm{~cm}$. The first continuum contour level at $3.6 \mathrm{~cm}$ is $0.75 \mathrm{mJy} /$ beam and increases by a factor of 1.3. For the H92 $\alpha$ line, the first contour is $20 \mathrm{Jy} /$ beam $\mathrm{m}$ $\mathrm{s}^{-1}$ and increases by a factor 1.4 . At $2 \mathrm{~cm}$, the first continuum contour is $0.5 \mathrm{mJy} /$ beam and the first line contour is $80 \mathrm{Jy} /$ beam m s$~^{-1}$. Both increase by a factor 1.5

In this paper, we present the first sub-arcsecond imaging of ionized gas in the nuclear region of NGC 253. The VLA was used in its A-configuration to observe the $\mathrm{H} 92 \alpha(8.3 \mathrm{GHz})$ and $\mathrm{H} 75 \alpha(15.3 \mathrm{GHz})$ radio recombination lines (RRLs) with angular resolutions of $0.5^{\prime \prime} \times 0.3^{\prime \prime}\left(\mathrm{PA}=0^{\circ}\right)$ and $0.3^{\prime \prime} \times 0.15^{\prime \prime}(\mathrm{PA}=$ $-20^{\circ}$ ) respectively. At the distance of $3 \mathrm{Mpc}$ to NGC $253,1^{\prime \prime}$ corresponds to a linear size of $16 \mathrm{pc}$. Since RRLs do not suffer from extinction, these observations are able to probe the ionized gas in the very central regions and have provided evidence for a possible SSC coincident with the central radio continuum peak.

\section{Observations and Results}

Observations were made in the largest (A) configuration of the VLA which has interferometric baselines ranging from $0.7-36.4 \mathrm{~km}$. For the H92 $\alpha$ observations near $8.3 \mathrm{GHz}$, a bandwidth of $25 \mathrm{MHz}$ and 31 spectral channels were used. The velocity resolution after hanning smoothing was $56 \mathrm{~km} \mathrm{~s}^{-1}$. For the $\mathrm{H} 75 \alpha$ line at $15.3 \mathrm{GHz}, 15$ spectral channels over a bandwidth of $50 \mathrm{MHz}$ with a resolution of $123 \mathrm{~km} \mathrm{~s}^{-1}$ was used. At both frequencies data was taken over two 8 hour sessions. Amplitude, phase and bandpass calibrations were made using standard methods. The continuum data was self calibrated and the solutions were applied to the line data. Natural weighting was applied to the visibilities to make the final continuum and line images. The rms noise in the line images were 80 $\mu \mathrm{Jy} /$ beam and $260 \mu \mathrm{Jy} /$ beam for the $\mathrm{H} 92 \alpha$ and $\mathrm{H} 75 \alpha$ lines respectively. 

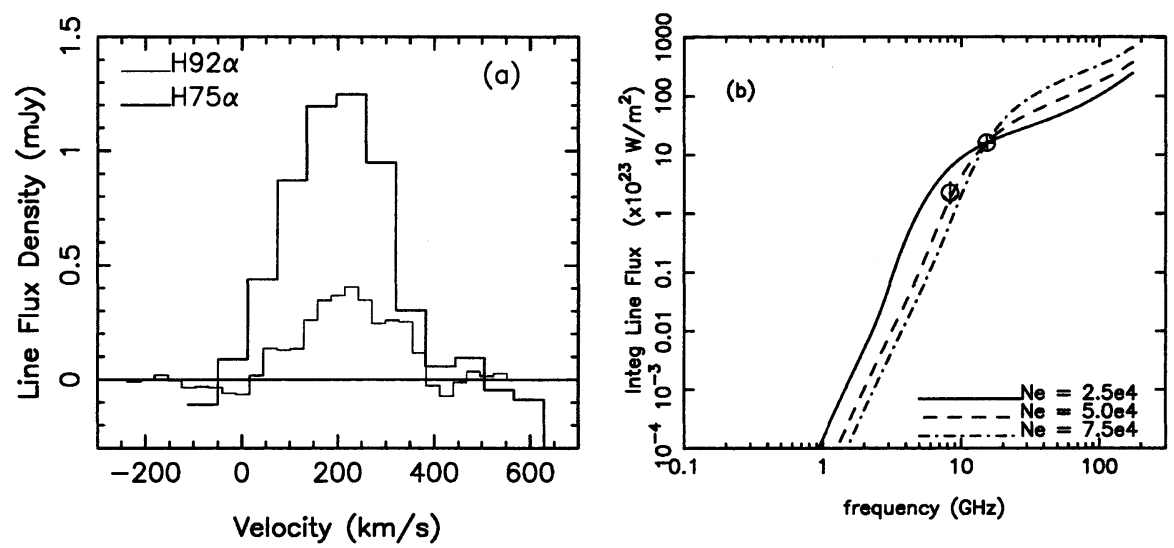

Figure 2. (a) Recombination line profiles at $8.3 \mathrm{GHz}$ and $15.3 \mathrm{GHz}$ at the position of the continuum peak at $2 \mathrm{~cm}$. (b) Model of RRL emission from a collection of HII regions at three different densities.

Fig 1 shows the line and continuum images of the central $5^{\prime \prime}$ region. The continuum images at $3.6 \mathrm{~cm}$ and $2 \mathrm{~cm}$ show compact structures which are consistent with the results obtained by Ulvestad \& Antonucci (1997). The grey scale with thick contours, superposed on the two continuum images, indicates the regions where RRL emission was observed. The H92 $\alpha$ line emission is sharply peaked at $\sim 0.8^{\prime \prime}$ south of the continuum peak and extends over a region of $\sim 2.5^{\prime \prime}(40 \mathrm{pc})$. The peak line emission is $1.2 \mathrm{mJy} /$ beam. Another weak compact line emission region is observed near a continuum peak in the southwest region along the major axis. The higher frequency $\mathrm{H} 75 \alpha$ line emission (Fig $1 \mathrm{~b}$ ) shows only two compact peaks near the central region. The brighter line emission is nearly coincident (offset $\sim 0.1^{\prime \prime}$ ) with the continuum peak at $2 \mathrm{~cm}$. The peak line emission is $1.5 \mathrm{mJy} / \mathrm{beam}$. In the high resolution observations at $2 \mathrm{~cm}$ any possible extended $\mathrm{H} 75 \alpha$ emission would be resolved out.

\section{Does the Ionized Gas Indicate a Super Star Cluster?}

In this short paper, we focus on the interesting implications of detecting a compact $\mathrm{H} 75 \alpha$ line emission region close to the radio continuum peak at 2 $\mathrm{cm}$. Fig $2 \mathrm{a}$ shows the $\mathrm{H} 75 \alpha$ line profile at the continuum peak $\left([\alpha, \delta]_{1950}=\right.$ $\left.00^{h} 49^{m} 5.793^{s},-25^{\circ} 33^{\prime} 39.06^{\prime \prime}\right)$ over one beam area $\left(0.3^{\prime \prime} \times 0.15^{\prime \prime}\right)$ corresponding to a linear size of $\sim 5 \mathrm{pc} \times 2.5 \mathrm{pc}$. The H92 $\alpha$ line profile at this position (shown in the same Fig) corresponds to a larger beam area $\left(0.5^{\prime \prime} \times 0.3^{\prime \prime}\right)$ and hence can be regarded as an upper limit to the H92 $\alpha$ line emission over the $5 \mathrm{pc} \times 2.5 \mathrm{pc}$ region. The velocity-integrated flux densities of the two lines in Fig 2a are 16.1 $\times 10^{-23} \mathrm{~W} \mathrm{~m}^{-2}$ and $2.3 \times 10^{-23} \mathrm{~W} \mathrm{~m}^{-2}$ respectively. Using these line strengths and also the observed continuum flux densities, we obtain constraints on the properties of the ionized gas in the central $5 \mathrm{pc} \times 2.5 \mathrm{pc}$ region.

Following the procedure described in Anantharamaiah et al (2000), the observed RRL emission is modeled as arising from a collection of $N$ HII regions 
characterized by density $n_{e}$, temperature $\mathrm{T}_{e}$ (assumed here to be $10,000 \mathrm{~K}$ ) and size $l$. Acceptable models are those which are consistent with the observed $\mathrm{H} 75 \alpha$ line, the upper limit to the H92 $\alpha$ line and the continuum flux densities at $8.3 \mathrm{GHz}, 15.2 \mathrm{GHz}$ and $22.3 \mathrm{GHz}$, which we estimate to be 55,41 , and 30 $\mathrm{mJy}$, respectively, using the high resolution images by Ulvestad \& Antonucci (1997). Fig $2 \mathrm{~b}$ displays three models with different densities and shows that, to be consistent with the $\mathrm{H} 92 \alpha$ line, only densities above $5 \times 10^{4} \mathrm{~cm}^{-3}$ are allowed.

The model that fits the data shows that within the $5 \mathrm{pc} \times 2.5 \mathrm{pc}$ region there are $35 \mathrm{HII}$ regions, each of density $7.5 \times 10^{4} \mathrm{~cm}^{-3}$ and size $0.25 \mathrm{pc}$. The volume filling factor of the ionized gas is 0.02 and the total mass is $530 \mathrm{M}_{\odot}$. The required Lyman continuum photon production rate, $\mathrm{N}_{\mathrm{Lyc}}$, is $1.2 \times 10^{52} \mathrm{~s}^{-1}$. Adopting the stellar atmosphere model of Vacca, Garmany, \& Shull (1996), this value of $\mathrm{N}_{\mathrm{Lyc}}$ corresponds to a total number of $\sim 10^{3} \mathrm{O}$ stars, assuming the Salpeter initial mass function and a stellar mass range of 1 to $80 \mathrm{M}_{\odot}$. The corresponding total stellar mass is $\sim 2 \times 10^{5} \mathrm{M}_{\odot}$ and the number of stars with $\mathrm{M}>1 \mathrm{M}_{\odot}$ is $7 \times 10^{4}$ and about $10^{6}$ stars with $\mathrm{M}>0.1 \mathrm{M}_{\odot}$. This high density of stars confined to a $5 \mathrm{pc} \times 2.5 \mathrm{pc}$ region correspond to that of a SSC (Meurer et al 1995). In NGC 253, four SSCs have been discovered in the nuclear region (Watson et al 1996) using HST observations. However, none of these are coincident with the radio continuum peaks. The detection of RRLs at sub-arcsecond resolution do indicate the possibility of an SSC near the central radio continuum peak which may be totally obscured at optical and infrared wavelengths.

The VLA is operated by the National Radio Astronomy Observatory which is a facility of the National Science Foundation operated under cooperative agreement by Associated Universities, Inc.

\section{References}

Anantharamaiah, K.R. \& Goss, W.M. 1996, ApJ, 466, L13

Anantharamaiah, K.R., Viallefond, F., Mohan, N.R., Goss, W.M., \& Zhao, J.H. 2000, ApJ, 537, 613

Arnaboldi, M., Capaccioli, M., Cappellaro, E., Held, E.V., \& Koribalski, B. 1995, AJ, 110,119

Boker, T., Krabbe, A., \& Storey, J.W.V. 1998, ApJ, 498, L115

Engelbracht, C.W., Rieke, M.J, Rieke, G.H., Kelly, D.M. \& Achtermann, J.M. 1998, ApJ, 505, 639

Keto, E., Hora, J.L, Fazio, G.G., Hoffmann, W., \& Deutsch, L. 1999, ApJ, 518, 183

Meurer, G.R., Heckman, T.M., Leitherer, C., Kinney, A., Robert, C., \& Garnett, D.R., 1995, AJ, 110, 2665

Prada, F., Gutierrez, C.M., \& McKeith, C.D. 1998, ApJ, 495, 765

Ulvestad, J.D. \& Antonucci, R.R.J. 1997, ApJ, 488, 621

Vacca, W.D., Garmany, C.D., \& Shull, J.M. 1996, ApJ, 460, 914

Watson, A.M., et al. 1996, AJ, 112, 534 